\title{
El campeonato de bertsolaris de Euskal Herria como institución canonizadora de las melodías, de los ejercicios y de las estrofas de bertsos*
}

\section{The Basque Country bertsolari Championship as a Canonizing Institution of Melodies, Exercises and Stanzas of bertsos}

ASIER BARANDIARAN AMARIKA

Facultad de Educación y Deporte - Hezkuntza eta Kirol Fakultatea

Euskal Herriko Unibertsitatea - Universidad del País Vasco

C/ Juan Ibáñez de Sto. Domingo, 1. Vitoria-Gasteiz, 01006

asier.barandiaran@ehu.eus

Orcid ID 0000-0002-0249-3246
Resumen: Las competiciones de bertsolaris tienen su culmen en la Euskal Herriko Bertsolari Txapelketa (Campeonato de Bertsolaris de Euskal Herria) cada cuatro años. Es un campeonato con diversas fases y con una gran final donde compiten ocho bertsolaris y donde se decide quién ostentará la txapela (el trofeo dado al ganador) durante los siguientes cuatro años. Se trata de un acto de canonización en donde repertorios de ejercicios, tipos de bertsos (métricas de estrofas) y melodías (algunas nuevas y otras "recuperadas de la periferia”) se muestran al gran público, son difundidas en los medios y son juzgadas por el jurado. Todo ello hace que algunas melodías, algunos ejercicios bertsolarísticos y algunas estrofas se incorporen al centro del sistema cultural del bertsolarismo. En este trabajo se intenta hacer un seguimiento de estos tres parámetros tomando como re- ferencia básica las últimas tres finales de bertsolaris (2009; 2013; 2017) y también se trata de constatar si esa canonización de la final del campeonato tiene alguna repercusión en sesiones de bertsolaris ordinarias o en campeonatos territoriales. Algunos artículos de personas estudiosas del bertsolarismo se utilizarán como información cualitativa que sostenga la explicación de los procesos de canonización en géneros orales improvisados como este, en donde los campeonatos, como institución, juegan un papel clave. El condicionante psicológico que la Txapelketa juega en el bertsolari y en el jurado también será inquirido, sopesado y valorado en este trabajo.

Palabras clave: Bertsolarismo. Txapelketa (Competición). Melodía. Estrofa. Canonización. Institución. Repertorio.

\footnotetext{
* Trabajo dentro del proyecto del Grupo Consolidado de Investigación LAIDA (Literatura eta Identitatea) que pertenece a la red de Grupos de Investigación del Gobierno Vasco (IT 1397/19) y está reconocido por la Universidad del País Vasco/Euskal Herriko Unibertsitatea (PPGA 20/19).
} 
Abstract: Bertsolaritza competitions have their climax in the Euskal Herriko Bertsolari Txapelketa (Bertsolaritza Championship of Euskal Herria) every four years. It is a championship with different phases and with a great final where eight bertsolari compete and where it is decided who will "reign" for four years, holding the txapela (the trophy given to the winner). This is an act of canonization where repertoires of exercises, types of bertso (stanza metrics) and melodies (some new and others "recovered from the periphery") are shown to the general public, disseminated in the media and judged by the jury. All this means that some melodies, some bertsolaristic exercises, and some stanzas are incorporated into the center of the cultural system of bertsolaritza. In this paper we follow up on these three parameters taking as a basic reference the last three bertsolari competition finals (2009; 2013; 2017), and also try to verify if this "canonization" of the final of the championship has any verification in sessions of ordinary bertsolari or in territorial championships. Some articles by scholars from the field of bertsolaritza will be used as qualitative information that supports the explanation of the processes of "canonization" in improvised oral genres such as this one, where championships, as an institution, play a key role. The psychological conditioning that the txapelketa plays in bertsolaritza and in the jury will also be examined, assessed and evaluated in this paper.

Keywords: Bertsolaritza. Txapelketa (Competition). Melody. Stanza. Canonization. Institution. Repertoire.

\section{INTRODUCCIÓN}

ए 1 sistema cultural, y dentro de él, el sistema literario se puede entender en su dinamismo, en su evolución y en sus relaciones con los sistemas culturales circundantes gracias a la teoría de los polisistemas y con ciertas herramientas creadas dentro de él, como el esquema que propone Itamar Even-Zohar (31), basado en el conocido esquema de Jakobson:

\section{Institución (contexto)}

Repertorio (código)

Productor (emisor), "escritor" -..-_-_-_-_._-_.-.- Consumidor (destinatario), "lector"

\section{Mercado (contacto/canal) \\ Producto (mensaje)}

En el sistema cultural vasco -me refiero a la cultura hecha en euskera (creadores como escritores, creadores en el ámbito de los medios de comunicación, cantantes, ipuin kontalariak o contadores de cuentos, actores de teatro...)- el bertsolarismo ocupa un lugar muy central en la actualidad. Pero eso no es algo que se haya conseguido de la noche a la mañana y mucho menos sin esfuerzo. Porque el bertsolarismo, al ser un género oral, improvisado y no tradicional, se 
desarrolla en la oralidad, ${ }^{1}$ y es bien sabido que recientemente en la cultura occidental se ha dado preponderancia a la cultura escrita (lo oral se ha convertido en periférico), especialmente en el mundo académico: "the relented dominance of textuality in the scholarly mind is shown by the fact that to this day no concepts have yet been formed for effectively, let alone gracefully, conceiving of oral art as such without reference, conscious or unconscious, to writing" (Ong 10).

\section{AUGE DEL BERTSOLARISMO Y LAS TXAPELKETAS (CAMPEONATOS)}

Ese acercamiento al centro del sistema cultural durante el siglo Xx, entre otras razones, ha sido gracias a que, por ejemplo, antes de la Guerra Civil, varios agentes-promotores culturales, entre ellos personas de formación académica, como Aitzol - Jose Ariztimuño (1896-1936) o Manuel Lekuona (1894-1987), teorizaron sobre el bertsolarismo y vieron en él un instrumento para llegar más a la comunidad lingüística vasca y concienciarla para que se involucre en el renacimiento cultural.

Más adelante, el artista Jorge Oteiza (1908-2003) publica una obra titulada Quousque tándem...! (1963), en la que dedica un capítulo al bertsolarismo, afirmando que el bertsolari es una de las personas que mejor tiene interiorizada el "alma vasca" y su expresión estética. Además de ser un artista reconocido internacionalmente por su aportación escultórica, Jorge Oteiza ha sido una referencia cultural vasca de primera magnitud, también por sus proyectos e ideas artísticas para Vasconia (tuvieran o no éxito en términos de culminación física), y su prestigio ha supuesto que su influencia en el terreno de las ideas artísticas haya sido grande.

Años más tarde, gracias a la creación de la asociación de bertsolaris, con el nombre Euskal Herriko Bertsozale Elkartea (Barandiaran Amillano 25-52), se ha dado un impulso importante a este género oral, aunque aquí no analizaremos la aportación de dicha institución. La presencia en los medios de comunicación y, sobre todo, en la televisión pública vasca (ЕІTB) a principios de la década de los 90, también supuso una visibilidad que añade prestigio a este género que llega a convertirse casi en movimiento cultural. La presencia en el sistema educativo vasco también es otro hito importante por acceder a una

1. "Basque bertsolari, who is concerned with very modern, contemporary events and problems, is not recreating or retelling anything. His song is total improvisation" (Armistead/Zulaika 12). 
institución vital del sistema cultural que sostiene a la comunidad lingüística vasca (Barandiaran Amarika).

Por otra parte, los mismos bertsolaris a veces se han autogestionado la práctica del bertsolarismo con escuelas de bertsolaris o "bertso-eskolak" en donde, de manera semi-formal, se practican y aprenden algunas técnicas y aspectos formales del bertsolarismo y se simulan sesiones ante un público imaginario. Aunque en la actualidad se usan cada vez más recursos on-line y nuevas tecnologías, históricamente en las "bertso-eskolak" se ha combinado la escritura y la lectura (manuales con ejercicios, diccionarios de rimas, lectura cantada de bertsos antológicos); es decir, a través de técnicas transicionales, con la audición y la improvisación oral. En el año 1959 se crea la primera bertso-eskola en Bizkaia, en la localidad de Muxika (Enbeita 85-90), pero es sobre todo a partir de la década de 1980 cuando se empiezan a extender y formalizar. En la actualidad la base de datos de la Asociación de Bertsozales aporta una cifra total de 211 bertso-eskolas en todos los territorios de Euskal Herria. ${ }^{2}$

Todo ello ha hecho que los estudios o encuestas socioculturales recientes indiquen que el bertsolarismo es muy conocido y valorado por la comunidad lingüística vasca (Garzia 12-13; Garzia/Sarasua/Egaña 36-37), siendo sus consumidores no solo los que asisten a las sesiones (más de mil al año, sean campeonatos o no), sino los que ven o escuchan programas sobre ello en los medios (televisión, radio, cine, internet), los que participan en las escuelas de bertsolaris, los que reciben instrucción en el sistema reglado (en educación primaria y secundaria, principalmente): todos ellos son consumidores directos y, a veces, también indirectos. Casi todos los miembros de la comunidad lingüística vasca son, de un modo u otro, consumidores del bertsolarismo:

To begin with, the direct consumption of integral texts has been, and remains, peripheral to the largest part of "direct", let alone "indirect", consumers of "literature". All members of any community are at least "indirect" consumers of literary texts. In this capacity we, as such members, simply consume a certain quantity of literary fragments, digested and transmitted by various agents of culture and made an integral part of daily discourse. (Even-Zohar 36)

En la cultura vasca, el bertsolarismo ha llegado a ser un movimiento sociocultural (Zubiri), autoorganizado y con capacidad de adaptarse a los cambios sociales.

2. https://bdb.bertsozale.eus/es/web/bilaketa/index?\&fq[=mota_facet:\%22bertso_eskola $\% 22$. 
Otro agente importante han sido los campeonatos (en euskera, txapelketak). En este trabajo no voy a hacer una historia de los campeonatos (un resumen en castellano está en la página web de la Asociación de Bertsozales ${ }^{3}$ ), pero es sabido que, de alguna manera, siempre han estado presentes en el bertsolarismo (por su carácter dialógico y de desafío retórico). En general, los campeonatos han ido en la línea de acrecentar el prestigio social de este género oral, y cohesionar ciertos discursos culturales que se creaban en la lengua vasca: "Neurri batean, horretarako ere balio duelako Txapelketak: euskaltzaleengan diskurtso komun bat ezartzeko, Txapelketta aurreko gure jendartean dauden tentsio batzuk askatzeko. Baita beste batzuk sortzeko ere" (Mantxola 101 ['En cierta medida para eso también vale la Txapelketa: para implementar un discurso común entre los vascófilos, para liberar algunas tensiones previas de nuestras gentes antes de los campeonatos. Y para crear otras tensiones también']).

Aunque antes del siglo xx los bertsolaris, al moverse en el mundo de la oralidad, quedaban por debajo del radar de la cultura dominante, a modo de concurso, o reto, se documentan varios desafíos de bertsolaris en el siglo XIX (Azurmendi 150). Volviendo a mencionar el principio del siglo XX, antes de la Guerra Civil a los campeonatos/txapelketas se les llamaba bertsolari guduak ('guerras', 'batallas') y se buscaba a propósito (por los agentes culturales de entonces) que fuesen en lugares de prestigio como el teatro Victoria Eugenia de San Sebastián, por citar un lugar canonizador.

Siguiendo con el lugar de los campeonatos, es significativo que las últimas finales de las txapelketas hayan sido en el Bilbao Exhibition Center, edificio emblemático de un País Vasco en renovación de sus infraestructuras, económicas, sociales, etc., un edificio puntero tecnológicamente que es capaz de albergar grandes eventos con mucho público; y es que la txapelketa se ha convertido en un gran evento, que acoge a unas 14000 personas durante ocho horas de actuación de bertsolaris (además de las que siguen el evento en directo a través de los medios de comunicación).

El eco mediático que tienen las txapelketas, tanto las territoriales como la "nacional" (la de Euskal Herria) hace que todo lo que se haya preparado durante las eliminatorias previas y en la final tenga mucho impacto en el imaginario colectivo, en especial de la cultura vasca que vive en euskera.

Por eso, es de suponer o, con otras palabras, se podría formular la hipótesis de que el utilizar ciertas melodías en las finales de los campeonatos pue-

3. https://www.bertsozale.eus/es/bertsolaritza/historia/campeonatos?set_language=es. 
den otorgar un valor canónico precisamente a esas melodías y, por lo tanto, que tengan una influencia psicológica en los bertsolaris que actúan durante el año en sesiones ordinarias (es decir, fuera de los campeonatos). Por lo tanto, se pueden constituir en textos o plantillas transicionales para otras sesiones de bertsolaris. De hecho, las melodías los bertsolaris actuales ${ }^{4}$ las han aprendido usando textos escritos y escuchando y después cantando o repitiendo bertsos antológicos o bertsos (textos) que hicieron famosas esas melodías. Los bertsolaris actuales, por lo tanto, también en lo concerniente al uso de las melodías, parece que viven en una suerte de oralidad secundaria (Ong 11).

Empezando desde la segunda mitad del siglo Xx, los campeonatos "nacionales" han sido en los años que se citan y con los ganadores que se detallan a continuación:

\begin{tabular}{l|l} 
Organizados por Euskaltzaindia & Organizados por la Bertsozale Elkartea \\
\hline 1960 - Donostia - Iñaki Eizmendi "Basarri” & 1986 - Donostia - Sebastian Lizaso \\
1962 - Donostia - Manuel Olaizola "Uztapide” & 1989 - Donostia - Jon Lopategi \\
1965 - Donostia - Manuel Olaizola "Uztapide" & 1993 - Donostia - Andoni Egaña \\
1967 - Donostia - Manuel Olaizola "Uztapide" & 1997 - Donostia - Andoin Egaña \\
1980 - Donostia - Xabier Amuriza & 2001 - Donostia - Andoni Egaña \\
& 2005 - Barakaldo - Andoni Egaña \\
& 2009 - Barakaldo - Maialen Lujanbio \\
& 2013 - Barakaldo - Amets Arzallus \\
& 2017 - Barakaldo - Maialen Lujanbio \\
\hline
\end{tabular}

En este estudio preliminar me acercaré a las tres últimas txapelketas y a su poder canonizante en la actividad bertsolarística.

Las tres últimas "txapelketa nagusiak" son, como se ha señalado antes (con sus ganadores):

$$
\begin{aligned}
& 2009 \text { - Barakaldo - Maialen Lujanbio } \\
& 2013 \text { - Barakaldo - Amets Arzallus } \\
& 2017 \text { - Barakaldo - Maialen Lujanbio }
\end{aligned}
$$

De esas txapelketas tomaré como primera muestra las novedades en cuanto las melodías utilizadas. Pero para empezar, a modo de justificación, hay que seña-

4. Se entiende por "bertsolari actual" aquel que ha participado en las últimas tres competiciones de Euskal Herria. 
lar que la txapelketa del 2009 es especialmente significativa por varios motivos: porque supuso el final de la "Era Egaña" (4 txapelketas ganadas), porque por primera vez la participación femenina fue notable y porque, de hecho, una mujer gana la txapelketa nagusia por primera vez en la historia del bertsolarismo. El poder canonizador que tiene la txapelketa hace más visible este punto de inflexión hacia el género femenino (Foley 2010) y canoniza una serie de elementos, entre los que está la melodía. También desplaza otros elementos que gozaban de una hegemonía en los años precedentes. Las dos txapelketas posteriores entran en el ámbito de este trabajo por ser las más recientes y porque continúan siendo unos hitos importantes en la historia reciente del bertsolarismo improvisado.

\section{DEFINICIÓN DE LA MELODÍA Y DE SU ASPECTO TRANSICIONAL}

En esta primera parte del estudio quiero prestar atención lugar al doinu, a la melodía del bertso. ¿Qué es melodía? Es la sucesión de sonidos de carácter musical utilizado para bertsos, que son ejecutados a capella. La melodía la utilizan los bertsolaris (inconscientemente muchas veces) para usar la métrica correcta y, con sus características evocadoras, también es utilizada para acompañar al mensaje textual, para potenciarlo y darle mayor expresividad (y así llegar mejor al público). Los nombres que en euskera recibe la melodía son varios, como señala Dorronsoro: "Doinu, aire, melodia, musika, errezitapen, kantu, abesti...”. La melodía es, junto con la métrica (neurria) y la rima (errima) uno de los tres aspectos formales que se enseñan en las escuelas de bertsolaris (Garzia/Sarasua/Egaña 81-99).

De alguna manera, las melodías son "fórmulas de la mente" que ayudan al bertsolari en su labor de estar componiendo al mismo tiempo que ocurre la performance, la actuación. Son además (las melodías) una especie de molde o frame (Pagán Cánovas/Antović 68) que sirve como plantilla para construir un discurso y acompañan (a veces con la repetición, otras con un tono propicio para el efecto final del bertso) a la técnica del bertsolarismo, la cual está enfocada al final del bertso, al último punto. Estos moldes, además, hacen de puente entre el mundo musical vasco y el mundo musical de la cultura occidental, como veremos más adelante, siendo este también otro aspecto transicional (parecido al que analiza Gintsburg 2017).

No está del todo claro que las fórmulas en el bertsolarismo sean del mismo tipo que de otras tradiciones. Por ejemplo, en la tradición eslava, estudiada por Milman Parry y otros, la representación oral "recreaba" historias 
transmitidas oralmente por antiguos guslaris. En el bertsolarismo, no se recrean historias pasadas: cada bertso es de nueva creación. En cambio, el público sí coopera en la creación, o "co-crea", pues acepta como reto muchas veces adivinar el final del bertso, además de repetirlo (cuando la melodía lo posibilite).

Esto es posible por dos razones: a) porque las rimas utilizadas forman un repertorio compartido más o menos conocido por el público y se sabe que cada "puntu" acabará en una rima de la misma categoría utilizada en el resto de la estrofa; b) porque la melodía conduce al bertsolari a cantar con un número de sílabas concreto en donde adecuará las palabras que va a utilizar.

Estas dos razones, junto con la lógica del discurso de cada bertso, pueden dar pistas al oyente de cómo acabará el bertso el bertsolari... Cuando se acierta, se genera una energía de "comunión" y un placer intelectual al haber adivinado el "acertijo" del final, que es donde suele recaer el golpe de efecto retórico del bertso (Garzia/Sarasua/Egaña 102). Pero eso es posible, porque existe un sistema formulario de la mente: "formulas in the mind", en la expresión acuñada por Linda White: "The melody is a vital factor, and it imposes constraints upon the language with regard rhythms and rhymes. [...] In the Basque bertso, it can also be said that the melody is the constant part of the song" (26869). Un sistema formulario para la composición que abarca las melodías (propiciadoras de la métrica y composición mirando al final del bertso), las rimas y el "ritmo compositivo":

If we stretch the definition of formula to include not only content but also the process of fulfilling a required rhythm, then we might be able to say that bertsolariak do employ "formulas of mind" when creating extemporaneous verses, even though these formulas are not phrases that are memorized or repeated to build a story. In other words, the process of fleshing out a melody with the requisite number of syllables might be a formula, albeit an unconscious one, employed by the bertsolariak in the making of bertsoak. (White 272)

Aparte de la melodía, el hecho de que pueda haber otros tipos de fórmulas en el bertsolarismo parece que todavía está por estudiar y demostrar. Por lo pronto, nos podemos remitir al análisis estadístico del grupo IXA en el que, entre otros aspectos, después de analizar estadísticamente el tipo de rimas utilizadas, concluyen que en el campeonato del 2013 las más utilizadas son: "ena", "era" y "eta", las mismas (más utilizadas) que en el campeonato del 2009. Y si- 
guiendo con datos estadísticos del 2013, los "pies" (oinak), más utilizados: "dira", "dago", "gehiago", "eta" y "begira" (Agirrezabal 374). Si son los más utilizados, quiere decir que se repiten: habría que estudiar si eso conlleva que las palabras previas a estos pies, también se repiten, formando combinaciones que puedan ser fórmulas.

En cualquier caso, y volviendo a las tonadas, una de las características de las melodías del bertsolarismo es que cada sílaba del texto oral corresponde a una nota de la melodía (Dorronsoro), lo cual hace que las melodías sean un instrumento que usa el bertsolari para adecuarse a la métrica. Por lo tanto, la melodía es una estructura "prefabricada", una especie de estructura transicional entre la intencionalidad del discurso (en la mente del bertsolari) y su ejecución final (oral) en una sesión de bertsos improvisados. Por otra parte, el bertsolarismo tiene un público más o menos fiel que comparte muchos conocimientos con el bertsolari al ser el este una persona normal y corriente que convive (es de un estrato social igual) con su público. Parte de ese conocimiento compartido es el uso de las melodías y tonadas: el público implícitamente acepta el "juego" de que el mensaje será transmitido y recibido usando una melodía de un repertorio más o menos conocido. Ese repertorio es, además, una especie de "registro" compartido:

Registers in oral poetry work similarly. Each type of speech-act - whether it be bertsolaritza, Homeric epic, or verbal magic from the former Yugoslavia - has its own rules for composition (the performer's job) and reception (the audience's job), and each register is markedly different from the everyday discourse of informal talk. (Foley 2005, 73)

Citando a Juanito Dorronsoro, en la actuación del bertsolari la melodía se subordina a la palabra, pero la melodía es indispensable:

Esan izan da bertsoak bi alde dituela, hitza eta doinua; esan izan da bie$\tan$ garrantzitsuena hitza dela eta bigarrena haren zerbitzuan dabilela. Ez dut nik hori ukatuko, ezta gutxiago ere, baina mami-azal, kanpokobarrengo, zeresan-nolaesan eta antzeko bereizketa logiko horiek ez dakit zenbateraino diren errealitatearen adierazpen egokiak, ederraren mundua eta bapateko bertsogintza aztertzerakoan gehienbat. Nik beti azpimarratu izan dut, kantuz sortzen duela bertsolariak, kantuz jasotzen duela entzuleak eta kantuz irauten duela bertsozalearen gogoan eta oroimenean. Hiru alderdiok dira beraz baztertu ezinekoak doinuaren eginkizuna aztertzerakoan. 
[Se ha dicho que el bertso tiene dos caras, la palabra y la melodía; se ha dicho que entre las dos la palabra es la más importante y que la segunda está en servicio de aquella. No lo voy a negar, ni mucho menos, pero dicotomías "lógicas" como cogollo/piel, externo/interno, qué-decir/cómodecir y algunas parecidas no sé hasta qué punto son expresiones adecuadas a la realidad. Yo siempre he dicho que el bertsolari crea cantando, que el oyente recibe cantando y que cantando continúa en el interior y en la memoria del bertsozale. Los tres aspectos son insoslayables al analizar el quehacer de la melodía.]

Nos podríamos extender mucho en ciertos aspectos de la melodía. Cabe señalar que actualmente la Bertsozale elkartea después de un trabajo de investigación y de clasificación, teniendo en cuenta las características de las melodías y cómo han sido utilizadas (con qué temas y en qué contextos) propone la siguiente tipología de melodías:

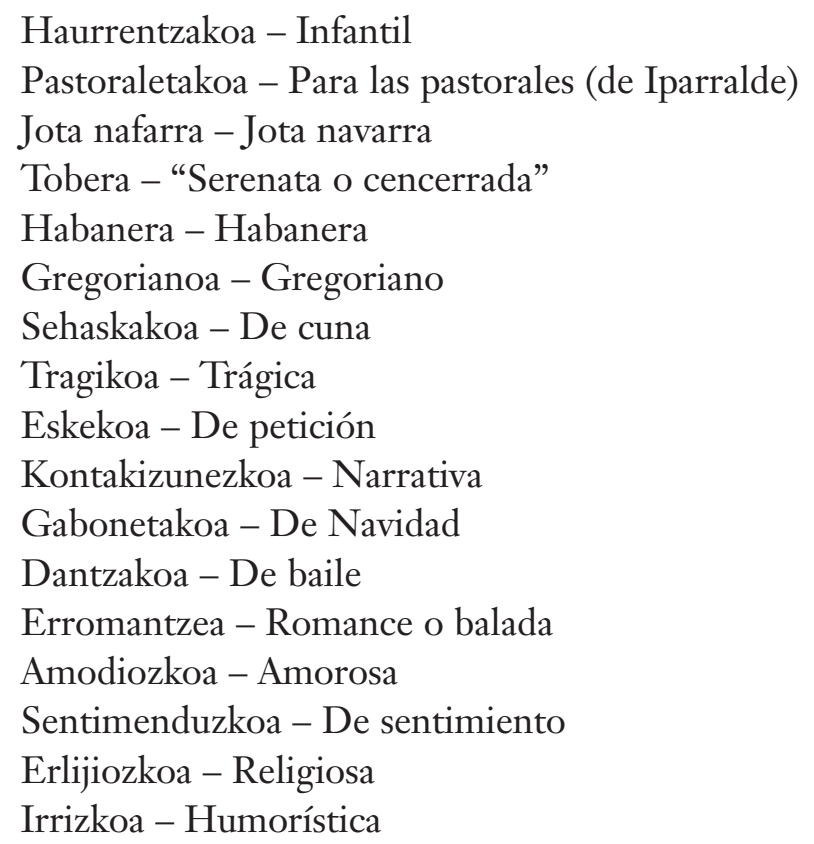

Dentro de esas 17 categorías, tenemos en el bertsolarismo más de 3000 melodías. Por lo tanto, el bertsolari, en función del tema sobre el que tiene que cantar o la situación comunicativa que se le presenta, tendrá que elegir una melodía o acordarla con el compañero de bertso (liro la liro): 
Bertsogintzaren aldetik begiratuta guztiok dakigu bertsoa egoki neurtzeko tresna dela doinua bertsolariarengan. Eginkizun apartekoa du beraz, zeren neurketa baita artegintza honetan lanbiderik menderagaitzena. Baina ez da kanpoko tresna garrantzitsu soil bat; hori baino sakonagora joaz, bertso izatearen muin muinean dakust kantua. Ez da ere, aurrena bertsoa moldatu eta gero doinuz jantzi; sorkuntza une berean han ari da airea. Bertsominetan dabilen artista popularrak kantuaren baitan uzten du bere sormena eta haren hegaletan doa bere barrena isurtzen sorkuntza une latz pozgarrian, eta horrela doinuz jaio da bertsoa mundura; kantuaren bidez, doinuarekin batera, doinu da bera oso osorik. Bertso eta doinu, bereiztu ezineko izakera bat berekoak, elkarrekin eta elkarrenganako eginak; kantu berri berria, bizia, freskoa: bertsoa. (Dorronsoro)

[Desde el bertsolarismo todos sabemos que la melodía es, para el bertsolari, un instrumento adecuado para medir. Por lo tanto, tiene una función especial, pues la métrica tiene en este arte el trabajo más difícil de dominar. Pero no es un instrumento exterior; yo veo al canto, yendo a lo más profundo, en lo más íntimo de la esencia del bertso. Tampoco debería ser, primero componer el bertso y luego vestirlo con la melodía; en el mismo momento de su creación está la melodía. El artista popular que está pariendo el bertso deja su producto creativo en el canto y en sus alas continúa vertiendo su interior en el momento áspero y gozoso de la creación, y así nace al mundo el bertso con su melodía; por medio del canto, junto con la melodía, en sí es una melodía en su totalidad. Bertso y melodía, entidad inseparablemente única, hechos juntos y el uno para el otro; canto nuevo, vivo, fresco: el bertso.]

$\mathrm{Y}$ es que, como los propios bertsolaris afirman, la melodía tiene influencia en el pensamiento y en el acto de recepción del acto comunicativo que es una sesión de bertsolaris.

En 1995 Juanito Dorronsoro (entendido en música y bertsolarismo) llegó a registrar, como se ha dicho, 3000 melodías utilizadas en el bertsolarismo (las describió con pentagramas y les puso nombre). De ellas, unas 2500 tienen un origen popular: en cantos folklóricos, religiosos, etc. El resto han sido compuestas por los propios bertsolaris o por músicos a petición de los bertsolaris, o han sido tomadas de canciones en otras lenguas.

Si nos fijamos en un aspecto del bertso, la melodía, recabamos los siguientes datos. El bertsolari y experto en música Martin Aramendi Arana (439-46) hace un recuento de las melodías utilizadas en las txapelketas (campeonatos) principales, con el siguiente resultado: 


\begin{tabular}{c|c} 
AÑo & NúMERO DE DOINUAK O MELodíAs \\
\hline 1967 & 20 \\
\hline 1980 & 38 \\
\hline 1982 & 73 \\
\hline 1986 & 136 \\
\hline 1989 & 156 \\
\hline 1993 & 109 \\
\hline 1997 & 105 \\
\hline 2001 & 103 \\
\hline 2005 & 110 \\
\hline 2009 & 105 \\
\hline 2013 & 118 \\
\hline 2017 & 161 \\
\hline
\end{tabular}

Una de las causas que puede justificar la subida en número en los años 80 y 90 es que, en esas décadas, se concedía un premio al bertsolari que más melodías utilizara. En este sentido, una vez más la institución vuelve a jugar un papel importante en la promoción del repertorio. Utilizamos "institución" en el sentido de Even-Zohar:

The 'institution' consists of the aggregate of factors involved with the maintenance of literature as a socio-cultural activity. It is the institution which governs the norms prevailing in this activity, sanctioning some and rejecting others. Empowered by, and being part of, other dominating social institutions, it also remunerates and reprimands producers and agents. As part of official culture, it also determines who, and which products, will be remembered by a community for a longer period of time. (37)

Después del pico en número de melodías de las txapelketas de 1986 y 1989, el número se moderó hasta la última, en la que se dio el récord de 161 melodías. 
NuEVAS MELODÍAS OCUPAN EL CENTRO DEL SISTEMA CULTURAL DEL BERTSOLARISMO

La tendencia a estrenar melodías nuevas ha seguido en los últimos 3 campeonatos (en los que nos queremos fijar un poco más). En el campeonato del 2009 se estrenaron 16. En el del 2013, seis. En el del 2017, el último, 23, más que nunca.

La institución hegemónica en este sistema cultural, la asociación de bertsolaris (Bertsozale elkartea), se ocupó de que en la década de los 80 y 90 se premiara (con un premio especial) al bertsolari que utilizara mayor variedad de melodías. Eso hizo que se activara el repertorio de melodías y se diera impulso a su divulgación.

El consumidor del bertso valorará también ese esfuerzo o esa aportación, aunque su valoración sea difícil de medir cuantitativamente. En todo caso, para los consumidores es vital que los bertsolaris sigan recurriendo a la tradición del doinutegi, del repertorio de tonadas utilizadas en el bertsolarismo y transmitidas de generación a generación y actualizadas en cada sesión o performance:

The mere fact of performance means that a speaker must be understood differently, and that an audience must adjust its reception to understand him or her accordingly. Tradition is the background or referent for the event; it "fills in the blanks" of each performance by relating what is happening in this performance to the audience's larger experience. (Foley 2005, 80)

Aunque eso no supone una cerrazón a la incorporación de nuevas melodías de origen no vasco: el hecho de que procedan de otras tradiciones musicales o culturales, sigue actuando como correa de transmisión, se sigue construyendo con "mimbres" reconocibles.

El hecho de que precisamente el campeonato sea el lugar para estrenar nuevas melodías es, parece ser, que consciente o inconscientemente se considera como un acto canonizador que atrae al centro del sistema cultural del bertsolarismo algunas producciones o repertorios que eran marginales, no porque con la celebración del campeonato se busque eso (los jueces no quieren premiar a la mejor melodía, ni al mejor modo de cantar el bertso, en general, sino que premian con su puntuación la calidad técnica y comunicativa en la actuación de ese preciso día), sino como consecuencia de su impacto mediático y por el prestigio que adquiere el ganador o ganadora. 
Martin Aramendi (442-45) hace una tipología de las nuevas melodías que se estrenan en los campeonatos:

1. Melodías compuestas por un músico específicamente para un bertsolari.

2. Melodías de un cantante adaptadas por los bertsolaris.

3. Melodías compuestas por un bertsolari.

En general, pocas veces una melodía compuesta por un músico para un campeonato en concreto tiene un éxito claro. En cambio, cuando la melodía utilizada es conocida por pertenecer a un cantante (vasco o de otra cultura) el hecho de que aparezca en el campeonato puede actuar de catapulta para aproximarse al centro del bertsolarismo o canonizarse. Vamos a dar algunos ejemplos, pocos, ya que la presente aportación no es más que el comienzo de una investigación.

\section{Txapelketa de 2009}

Con los versos del poeta Joxean Artze (1939-2018), Mikel Laboa publicó un disco en 1980 y tituló la canción "Martxa baten lehen notak". Maialen Lujanbio convirtió esta canción en un bertso de 16 puntos. Lo utilizó por primera vez en la txapelketa del 2009 en la eliminatoria de Bergara (7 de noviembre) (EHBE), en un ejercicio de la final y en los bertsos de despedida. En la base de datos de Xenpelar Dokumentazio Zentroa, que ha hecho pública en su página web, aparecen 25 resultados más como muestra, aunque no es una muestra exhaustiva.

La comisión que se dedica al seguimiento de las melodías ha catalogado a esta melodía como kontakizunezkoa, 'narrativa'. Quizá destaque en esa potencialidad, pero tiene también cierto aire épico e incluso emotivo. Parece que la emoción que rodeó a los bertsos de Maialen en Barakaldo, cuando por primera vez una mujer gana el campeonato de Euskal Herria supuso una canonización de la melodía y por ello su abundante uso. Cuantitativamente y cualitativamente se puede observar que esta melodía, después de ser cantada al final del campeonato del 2009, supuso un punto de inflexión en su canonización.

En el Campeonato de Bizkaia más de una vez ha aparecido esta melodía. El campeón de Bizkaia de 2016, Etxahun Lekue (Larrabetzu, 1980), la usó, como Maialen, en su último bertso de agradecimiento. En el campeonato de

5. https://bdb.bertsozale.eus/web/doinutegia/view/3211-eguzkiak-urtzen-du-han-goian-b. 
Bizkaia de 2018, la que fue campeona (también por primera vez, como Maialen, una mujer es campeona de Bizkaia en ese año), Onintza Enbeita (Muxika, 1979), utilizó esta melodía varias veces en ejercicios significativos (por ejemplo, en el llamado kartzelakoa, 'el de la cárcel', en donde tiene que cantar en solitario de modo más solemne sobre un tema propuesto). El recientemente doble campeón de Gipuzkoa, Beñat Gaztelumendi (Añorga, Gipuzkoa, 1987), lo ha utilizado en momentos clave del campeonato del $2019 .{ }^{6}$

\section{Txapelketa de 2013}

Melodía titulada "Gure herri ttiki hau da". " En la final del 2013 fue utilizada por Amets Arzallus (que terminaría siendo campeón) y por Aitor Sarriegi. Inspirada en una canción de la cantante Bebe, Xabier Silveira la modificó y la convirtió en bertso de 6 puntos (algunos bertsolaris lo usan como bertso de 8 puntos). Clasificada en el tipo "Kontakizunezkoa", narrativo.

Para ejercicios en solitario resultó ser muy adecuado por el tono que se crea con la melodía. Pero en este caso, esta melodía no hace más que reafirmarse en su hegemonía. La base de datos que publica Xenpelar arroja 25 resultados. Varios de ellos son posteriores al 2013, pero la mayoría son previos. Los primeros resultados datan del 2005: el primero, de Fredy Paia (Algorta, 1981), en Hendaia, dentro de una eliminatoria del campeonato de Euskal Herria de aquel año.

Txapelketa de 2017

Melodía "Kintak jarrita ondorenian". ${ }^{8}$ Un zortziko nagusia (de cuatro puntos), que ya fue utilizado por Txirrita antes de la Guerra Civil. Maialen lo recuperó para la txapelketa del 2017, pero hubo algunos cantantes que en la década de los 70 (1972, Julen Lekuona, Antton Valverde...) ya lo habían de alguna manera recuperado en un disco en donde se musicaban melodías antiguas (entre ellas, esta de José Manuel Lujanbio, Txirrita, 1860-1936). Hay que señalar que, aunque es una melodía muy buena para ejercicios en solitario, y que al oído del bertsozale es agradable, no ha tenido mucho impacto y en la base de datos de Xenpelar solo publican un resultado.

6. https://bertsoa.eus/doinuak/eguzkiak-urtzen-du-han-goian-b.

7. https://bdb.bertsozale.eus/web/doinutegia/view/3155-gure-herri-ttiki-hau-da.

8. https://bdb.bertsozale.eus/web/doinutegia/view/1497-kintak-jarrita-ondorenian-kubako-gerrarena. 
También hay que destacar la melodía titulada "Gaur udaberri bat dabil" inspirada en la canción "Papá, cuéntame otra vez" de Ismael Serrano. Oihana Iguaran la utilizó en una eliminatoria (en Leitza, Navarra) previa a la final de Barakaldo. En la versión de Oihana Iguaran, el bertso es de 6 puntos de 16 sílabas cada uno (divididos en hemistiquios de $8+8$ ). La misma bertsolari, quien ha "domesticado" esta melodía, aparece utilizándola en otras dos ocasiones según la base de datos accesible al público de Xenpelar Dokumentazio Zentrua. ${ }^{9}$

La melodía "Oraindik hango oihartzun hots bat", ${ }^{10}$ del cantante fallecido Imanol Larzabal (1947-2004), fue utilizada la primera vez por Maialen Lujanbio y Aitor Mendiluze en la final del 2017. Dicho sea de paso, y volviendo al esquema de Itamar Even-Zohar, en este caso el "productor" o el promotor de esta melodía no es un bertsolari sino, al menos, dos: Maialen y Aitor; aunque, no habría que descartar que el ya fallecido Imanol también es, sin duda, la causa inicial y por lo tanto también participara de ese acto "productor" o promotor (Even-Zohar 35). En la página web de Xenpelar solo aparece un resultado. En Bertsoa.eus, aparecen tres. Aunque ha tenido repercusión en los medios, ${ }^{11}$ parece que va a necesitar tiempo para afincarse y extenderse a otros bertsolaris.

En cuanto al campeonato del 2017, cabe indicar que, como antes se ha señalado, al presentar más melodías que todos los anteriores, también la tendencia a utilizar melodías de cantantes (vascos o no vascos) experimenta una eclosión. Se trata, en este caso, de una aportación de la periferia del sistema cultural vasco o de aportaciones de otras culturas al corazón del sistema cultural vasco, un movimiento centrípeto, que ha venido a enriquecer el doinutegi o repertorio de melodías del bertsolarismo. Vamos a mencionarlos:

- Melodía titulada "Egunkarian ikusi nuen". Inspirada en la canción "Ne me quitte pas" del belga Jaques Brel. La cantó Igor Elortza en el ejercicio en solitario llamado "kartzelakoa".

- Melodía titulada "Irun badaukazu zerbait". Inspirada en la canción "Paloma" del cantante argentino Andrés Calamaro. La cantó Aitor Sarriegi en tres ocasiones en el campeonato.

9. https://bdb.bertsozale.eus/web/doinutegia/view/3497-gaur-udaberri-bat-dabil.

10. https://bdb.bertsozale.eus/web/doinutegia/view/3491-oraindik-hango-oihartzun-hots-bat.

11. Por ejemplo, en la radio pública vasca en euskera, dedicaron tiempo a esta elección de melodía: https://www.eitb.eus/eu/irratia/euskadi-irratia/programak/hitza-jolas/audioak/osoa/5917333/ audioa-mendiluze-eta-lujanbio-bake-artisauen-aire-berria------------/. 
- Melodía titulada "Kirolarion bizitza dugu". Inspirada en la canción "La Voz" de la cantante catalana Rosa Zaragoza. Fue utilizada (con alguna modificación) por la bertsolari Nerea Elustondo.

- Melodía titulada "Haur bat izateko nahia". Inspirada en la canción "Negras sombras" de la cantante gallega Luz Casal y utilizada por Jon Martin por primera vez en un ejercicio en solitario.

- Melodía titulada "Pertsonok ez al daramatzagu". Inspirada en la canción "Itzaltzuko Bardo" del cantante oriotarra Benito Lertxundi y utilizada por el bertsolari lapurtarra Sustrai Colina en un ejercicio en solitario en la final, pero Oihana Iguaran también la utilizó en una eliminatoria anterior.

- Melodía titulada “Agure zahar batek zion”. Inspirada en la canción del catalán Lluis Llach titulada "L'estaca", cantada por Jon Martin en el bertso de saludo de la semifinal de Segura, en vísperas del 1 de octubre, fecha en que se celebró un referéndum en Cataluña sobre la independencia. El día siguiente, también hubo otra semifinal en Leitza y los bertsolaris hicieron referencias a Cataluña en los bertsos iniciales de saludo.

Se trata de una tendencia que, creemos, eclosiona o se consagra en el campeonato del 2017, pero que tiene su origen (en sentido ascendente) en años anteriores. El primer bertsolari que empezó a utilizar una melodía no vasca en un campeonato fue Jon Maia en el campeonato de 1997 al servirse de la canción "Camarón" del grupo Pata Negra en un bertso de saludo. En el 2001 Igor Elortza se sirvió de la canción "El desaparecido" del cantante Manu Chao, y Xabier Silveira en el 2005 hizo lo propio con "Razones" de la cantante Babe. Fuera de los campeonatos, el mismo Jon Maia desde el año 2006 empieza a amoldar melodías de los cantantes Bob Marley y Bob Dylan ("Redemption Song”, del primero y "Blowin' in the Wind", del segundo) y más tarde esas mismas melodías harán acto de presencia en los campeonatos del 2009, a cargo de los bertsolaris Xabier Paya y Ander Oleaga (Aramendi 442).

\section{LA PLAZA PRESIONA HACIA EL CENTRO}

Hasta ahora nos hemos centrado en cómo el centro influye en la periferia, siendo el centro o un centro la txapelketa, y la periferia de las performances bertsolarísticas, las sesiones ordinarias que a lo largo del año se celebran sin carácter competitivo ("la plaza"). 
Pero la tensión o la influencia es en ambos sentidos. Es decir, la plaza -por nombrar de alguna manera el quehacer bertsolarístico que no está en el centro o corazón de la actuación canonizadora-, la plaza, decimos, tiene también su fuerza y su prestigio. Se puede observar que algunos ejercicios como la "sesión por puntos" (acuñada en euskera con el sintagma "puntukako saioa"), es decir, la sesión en la que los bertsolaris se turnan cantando un solo punto hasta terminar el bertso, que son muy bien acogidos en las plazas, han pasado a ser parte de los ejercicios de los campeonatos (en los últimos dos campeonatos). Ya desde el campeonato del 2005 se introduce este ejercicio en la final (en ediciones anteriores no se realizó este ejercicio) y lo tendremos en las tres finales objeto de este análisis.

En el ejercicio por puntos turnados, por ejemplo, se introduce o se propone, además, un tema humorístico, que crea distensión en la final. Esto también parece que es una influencia de la plaza. Los bertsolaris se entrenan durante el año para que con estos temas también el público se entretenga y se relaje tras la escucha de otros temas más dramáticos o de más gravedad.

\section{ALgUNAS CONCLUSIONES}

Sin ánimo de agotar todas las conclusiones que se pueden colegir de lo anteriormente analizado, sabiendo que este análisis se puede extender en su objeto de estudio, se pueden mencionar las que nos parecen más claras y evidentes:

- La txapelketa tiene sin duda un poder canonizador de las melodías, dándolas a conocer, extendiendo su uso y prestigiando unos tipos de medidas.

- Pero el poder canonizador tiene también sus límites, porque solo en ciertos momentos y con ciertos temas se puede llegar a impactar en el aficionado y, sobre todo, en el compañero de bertso. Una melodía que en la txapelketa ha funcionado bien, puede no tener continuidad en la plaza, por no haberse cantado en un momento mágico. Una muestra más de que el bertsolarismo tiene mucho de arte e inspiración, más que de objetividad.

- La txapelketa es un acto canonizador de facto (no por ser buscado), pero la plaza es el contrapunto que rivaliza con la txapelketa. Una consecuencia es que varios ejercicios y varios tipos de temas (y tonos) se han ido incorporando a la txapelketa con buen resultado, porque en la plaza ya de hecho tienen buen resultado. 
- Lo anteriormente expuesto tiene que ser sistematizado y pormenorizado. Un muestreo más amplio también sería necesario para hacer las inferencias más precisas y fiables. Estas líneas de investigación necesitan contrastarse con un corpus más exhaustivo y pueden ampliarse a otros aspectos, como el modo de encarar los temas, la tipología de temas propuestos (por los gaijartzailes, los que presentan y dicen el tema al bertsolari) en los campeonatos y la canonización de los más adecuados, la repercusión de haber llegado a la final y su consecuencia en número de actuaciones a las que son convocados después, etc.

\section{OBRAS CITADAS}

Agirrezabal, Manex et al. "Bertsolari Txapelketa Nagusien analisi estatistikoa”. Bertsolari Txapelketa Nagusia 2013. Ed. Euskal Herriko Bertsolari Elkartea. Oiartzun: Lanku Bertso Zerbitzuak, 2014. 373-77.

Aramendi, Martin. "Gaur udaberri bat dabil". Euskal Herriko Txapelketa Nagusia 2017. Ed. Euskal Herriko Bertsolari Elkartea. Oiartzun: Lanku Bertso Zerbitzuak, 2018. 439-46.

Armistead, Samuel G., y Joseba Zulaika, eds. Voicing the Moment: Improvised Oral Poetry and Basque Tradition. Reno: Center for Basque Studies, University of Nevada, 2005.

Azurmendi, Joxe. "Bertsolaritzaren estudiorako". Jakin 14-15 (1980): 139-64. Barandiaran Amarika, Asier. "Bertsolaritza eskolan izeneko programa Nafarroako Lehen Hezkuntzan: onurak eta erronkak hizkuntzaren jabekuntzari begira”. Horizontes científicos y planificación académica de lenguas y literaturas. Eds. Xaquin Nuñez et al. Ribeirao: Ediçoes Humus, 2015. 245-60.

Barandiaran Amilano, Alberto. Beste larogei urtian. Donostia: Euskal Herriko Bertsozale Elkartea, 2011.

Dorronsoro, Juanito. Bertso doinutegia, 1995. <https://bdb.bertsozale.eus/orriak/ get/5-ataleko-informazioa-bertso-doinutegia\#sarrera>.

Enbeita, Jon. Enbeitatarrak III. Oiartzun: Sendoa, "Txirrita Saila”, 2000.

Euskal Herriko Bertsozale Elkartea. Bertsolari Txapelketa Nagusia 2009. Oiartzun: Lanku Bertso Zerbitzuak, 2010.

Euskal Herriko Bertsozale Elkartea. Bertsolari Txapelketa Nagusia 2013. Oiartzun: Lanku Bertso Zerbitzuak, 2014.

Euskal Herriko Bertsozale Elkartea. Bertsolari Txapelketa Nagusia 2017. Oiartzun: Lanku Bertso Zerbitzuak, 2018. 
Even-Zohar, Itamar. "Factors and Dependencies in Culture: A Revised Outline for Polysystem Culture Research". Canadian Review of Comparative Literature 24.1 (1997): 15-34.

Foley, John Miles. "Comparative Oral Traditions". Voicing the Moment: Improvised Oral Poetry and Basque Tradition. Eds. Samuel G. Armistead y Joseba Zulaika. Reno: Center for Basque Studies, University of Nevada, 2005. 65-81.

Foley, John Miles. "2009ko Bertsolaritza Txapelketa”. Bertsolari Txapelketa Nagusia 2009. Ed. Euskal Herriko Bertsozale Elkartea. Oiartzun: Lanku Bertso Zerbitzuak, 2010. 301-10.

Garzia, Joxerra. Bertsolaritza. El bertsolarismo. Colección Cultura Vasca, 9. San Sebastián: Etxepare Euskal Institutoa, 2012.

Garzia, Joxerra, Jon Sarasua y Andoni Egaña. Bat-bateko bertsolaritza. Gakoak eta azterbideak. Donostia: Bertsozale Elkartea, 2005.

Gintsburg, Sarali. "It's got some meaning but I am not sure... The role of the particle ( $w a)-m a$ in the oral and transitional poetry of the Jbala (northern Marocco) from the congnitive perspective". Pragmatics of Cognition 24.3 (2017): 474-95.

Mantxola, Manex. "BECeko arratsaldeko kronika-edo". Bertsolari aldizkaria 109 (2018): 81-106.

Ong, Walter. Orality and Literacy: The Technologizing of the Word. New York: Methuen, 1982.

Pagán Cánovas, Cristóbal, y Mihailo Antović. "Formulaic creativity: Oral poetics and cognitive grammar". Language \& Communication 47 (2016): 66-74.

White, Linda. "Formulas in the Mind: A Preliminary Examination to Determine if Oral Formulaic Theory May Be Applied to the Basque Case". Voicing the Moment: Improvised Oral Poetry and Basque Tradition. Eds. Samuel G. Armistead y Joseba Zulaika. Reno: Center for Basque Studies, University of Nevada, 2005. 265-80.

Zubiri, Harkaitz. "Hirugarren industria iraultzako bertsolaritza". Bertsolari aldizkaria (2019): 8 de diciembre de 2019. <https://www.bertsolari.eus/ erreportajeak/harkaitz-zubiri-hirugarren-industria-iraultzako-bertsolaritza/>. 